\title{
Generalized Eigenfunction Method for Floating Bodies
}

\author{
Michael H. Meylan Colm Fitzgerald \\ Department of Mathematics, University of Auckland, New Zealand \\ e-mail addresses: meylan@math.auckland.ac.nz, c.fitzgerald@math.auckland.ac.nz
}

\section{Introduction}

We present here a solution in the time domain using the generalized eigenfunction method for floating bodies which allows the solution for a given initial displacement (and initial velocity) to be computed from the frequency domain solutions driven by an incident wave. This method has been developed for rigid bodies by Hazard \& Lenoir (2002); Meylan (2009), for elastic bodies by Meylan (2002); Hazard \& Meylan (2007) and previously for floating bodies by Hazard \& Loret (2007). Note that this later paper was very theoretically focused and no numerical simulations were presented and the method was not developed with such calculations in mind. We present here a formulation derived for a numerical solution and some example numerical calculations.

\section{Initial value problem for a freely floating structure}

Consider a surface-piercing structure, constrained to move in heave and pitch, floating in an inviscid and incompressible fluid of constant finite depth $h$. The motion of the fluid is assumed to be irrotational and, along with the amplitude of the structure motion, sufficiently small so as to permit its description within the framework of the linearized water-wave theory. Attention is restricted to two dimensions and Cartesian coordinates $(x, z)$ are chosen with $z$ directed vertically up-wards and with the origin in the mean free surface. The floating body problem involves a dynamic coupling of the motion of the body and surrounding fluid. A full solution of the problem requires the simultaneous solution of the fluid motion, described by the velocity potential $\Phi$, and the body motion, described by the displacement $\xi(t)$. Only heave and pitch body motions are considered here so that the displacement is two-component vector.

The motion of the fluid is governed by
Laplace's equation subject to various boundary conditions on each enclosing surface. Therefore, velocity potential $\Phi$ satisfies Laplace's equation

$$
\nabla^{2} \Phi=0
$$

within the fluid and the bed condition

$$
\frac{\partial \Phi}{\partial n}=0 \quad \text { on } \quad z=-h .
$$

The free-surface elevation $\zeta(x, t)$ is related to $\Phi$ through the linearized free-surface conditions

$$
\frac{\partial \Phi}{\partial t}=-g \zeta \quad \text { on } \quad z=0,
$$

where $g$ is the acceleration due to gravity, and

$$
\frac{\partial \zeta}{\partial t}=\frac{\partial \Phi}{\partial z} \quad \text { on } \quad z=0 .
$$

The motion of the fluid is coupled to the motion of the structure by the boundary condition on the structure surface

$$
\frac{\partial \Phi}{\partial n}=\partial_{t} \xi_{3} n_{3}+\partial_{t} \xi_{5} n_{5} \quad \text { on } \quad \Gamma,
$$

with the body motions in heave and pitch determined by

$$
\begin{aligned}
& \left(\begin{array}{cc}
M & -M x^{c} \\
-M x^{c} & I_{M}
\end{array}\right)\left(\begin{array}{l}
\ddot{\xi}_{3} \\
\ddot{\xi}_{5}
\end{array}\right) \\
= & -\rho \int_{\Gamma} \Psi\left(\begin{array}{l}
n_{3} \\
n_{5}
\end{array}\right) d S-\rho g\left(\begin{array}{ll}
W & I_{1}^{A} \\
I_{1}^{A} & I_{W}
\end{array}\right)\left(\begin{array}{l}
\xi_{3} \\
\xi_{5}
\end{array}\right)
\end{aligned}
$$

where $x_{c}$ is the $x$ coordinate of the centre of mass and $I_{1}^{A}$ is the (non-dimensionalised) first moment of the waterplane. In this case where no assumptions have been made regarding the geometry of the body cross-coupling of the modes of motion (where motion in pitch influences motion in heave and vice versa) is possible. However, if the body is symmetric about the $z$-axis and the centre of mass and centre of rotation lie on the same central axis (it is assumed the centre of rotation lies at the origin on the free-surface) then $I_{1}^{A}=0$ and 
$x^{c}=0$ so that the equations for heave and pitch decouple and become

$$
\begin{aligned}
& \left(\begin{array}{cc}
M & 0 \\
0 & I_{M}
\end{array}\right)\left(\begin{array}{l}
\ddot{\xi}_{3} \\
\ddot{\xi}_{5}
\end{array}\right) \\
= & -\left\{\rho \int_{\Gamma} \Psi\left(\begin{array}{l}
n_{3} \\
n_{5}
\end{array}\right) d S+\rho g\left(\begin{array}{cc}
W & 0 \\
0 & I_{W}
\end{array}\right)\left(\begin{array}{l}
\xi_{3} \\
\xi_{5}
\end{array}\right)\right\} .
\end{aligned}
$$

The absence of coupling between modes results in a significantly simplified problem and we will focus on this case.

\section{Spectral formulation}

The introduction of the acceleration potential $\Psi=\partial_{t} \Phi$ allows, after some further manipulations, the floating body problem to be written in the form of an abstract wave equation. In the case of a floating body with a vertical axis of symmetry moving in both heave and pitch the boundary value problem is

$$
\begin{gathered}
\Delta \Psi=0 \quad \text { in the fluid, } \\
\partial_{n} \Psi=0 \quad \text { on } \quad z=-h \\
\Psi=-\zeta \quad \text { on } \quad \mathbf{x} \in F \\
\partial_{n} \Psi=-\left(\begin{array}{cc}
1 / m & 0 \\
0 & 1 / I_{m}
\end{array}\right) \times \\
\left\{\int_{\Gamma} \Psi\left(\begin{array}{l}
n_{3} \\
n_{5}
\end{array}\right) d S+\left(\begin{array}{cc}
W & 0 \\
0 & I_{W}
\end{array}\right)\left(\begin{array}{l}
\xi_{3} \\
\xi_{5}
\end{array}\right)\right\}\left(\begin{array}{ll}
n_{3} & n_{5}
\end{array}\right)
\end{gathered}
$$

and

$$
\begin{gathered}
\partial_{t}^{2} \zeta=\partial_{n} \Psi, \quad \mathbf{x} \in F, \\
\partial_{t}^{2} \xi=\mathcal{P} \partial_{n} \Psi, \quad \mathbf{x} \in \Gamma,
\end{gathered}
$$

respectively, where $\mathcal{P}$ is a projection operator which gives the $n_{3}$ and $n_{5}$ components of the normal velocity on the body.

For the spectral formulation we write the evolution equation as

$$
\partial_{t}^{2}\left(\begin{array}{l}
\zeta \\
\xi
\end{array}\right)+\left(\begin{array}{ll}
\mathcal{A}_{11} & \mathcal{A}_{12} \\
\mathcal{A}_{21} & \mathcal{A}_{22}
\end{array}\right)\left(\begin{array}{l}
\zeta \\
\xi
\end{array}\right)=0
$$

where the Dirichlet-to-Neumann operators $\mathcal{A}_{12}$, $\mathcal{A}_{21}$ and $\mathcal{A}_{22}$ map a vector to a scalar, a scalar to a vector and a vector to a vector, respectively.
The operators $A_{11}$ and $A_{21}$ map in the following way:

$$
\begin{aligned}
& \mathcal{A}_{11}: \quad \zeta \rightarrow-\Psi_{n}(\mathbf{x}), \quad \mathbf{x} \in F, \\
& \mathcal{A}_{21}: \quad \zeta \rightarrow-\partial_{t}^{2} \xi
\end{aligned}
$$

where $\Psi$ is the solution of Laplace's equation, the zero velocity bed condition and the following free-surface and structure surface boundary conditions

$$
\begin{aligned}
\Psi & =-\zeta \quad \text { on } \quad \mathbf{x} \in F \\
\partial_{n} \Psi & =\left(\begin{array}{l}
-1 / m\left(\int_{\Gamma} \Psi n_{3} d S\right) \\
-1 / I_{m}\left(\int_{\Gamma} \Psi n_{5} d S\right)
\end{array}\right)\left(\begin{array}{ll}
n_{3} & \left.n_{5}\right) \quad \text { on } \quad \partial \Omega .
\end{array}\right.
\end{aligned}
$$

Similarly, the operators $A_{12}$ and $A_{22}$ map in the following way:

$$
\begin{array}{ll}
\mathcal{A}_{12}: & \xi \rightarrow-\Psi_{n}(\mathbf{x}), \quad \mathbf{x} \in F \\
\mathcal{A}_{22}: & \xi \rightarrow-\partial_{t}^{2} \xi,
\end{array}
$$

where in this case $\Psi$ corresponds to a solution of Laplace's equation with a zero normal velocity on the bed and a zero free-surface elevation boundary condition:

$$
\begin{aligned}
\Psi & =0 \quad \text { on } \quad \mathbf{x} \in F \\
\partial_{n} \Psi & =\left(\begin{array}{c}
-1 / m\left(\int_{\partial \Omega} \Psi n_{3} d S+W \xi_{3}\right) \\
-1 / I_{m}\left(\int_{\partial \Omega} \Psi n_{5} d S+I_{W} \xi_{5}\right.
\end{array}\right)\left(\begin{array}{ll}
n_{3} & n_{5}
\end{array}\right) \\
& \text { on } \quad \partial \Omega .
\end{aligned}
$$

The energy inner product of this matrix is best expressed in terms of the displacement vector

$$
\xi=\left(\begin{array}{l}
\xi_{3} \\
\xi_{5}
\end{array}\right)
$$

and the matrix of restoring coefficients

$$
\mathbf{W}=\left(\begin{array}{cc}
W & 0 \\
0 & I_{W}
\end{array}\right)
$$

as

$$
\begin{aligned}
\left\langle\left(\begin{array}{l}
\zeta \\
\xi
\end{array}\right),\left(\begin{array}{l}
\zeta^{\prime} \\
\xi^{\prime}
\end{array}\right)\right\rangle_{\mathcal{A}} & =\int_{F} \zeta \zeta^{\prime *} d x+\xi \cdot\left(\mathbf{W} \xi^{\prime}\right)^{*} \\
& =\int_{F} \zeta \zeta^{\prime *} d x+\xi_{3} W \xi_{3}^{\prime *}+\xi_{5} I_{W} \xi_{5}^{*}
\end{aligned}
$$

We can show that the evolution operator $\mathcal{A}$ in equation (9) is symmetric and we assume this implies self-adjointness. 
Solution as expansion in eigenfunctions

Given that the operator $\mathcal{A}$ is self-adjoint (in the Hilbert space defined by the inner product) then it possesses a continuous spectrum and its corresponding eigenfunctions are the frequencydomain solutions for the floating body waterwave problem. To fully describe the eigenfunctions it is necessary to specify the incident wave potential in addition to the frequency. This is because there are two eigenfunctions for every incident wave frequency, one corresponding to waves from the left $\kappa=1$ and one corresponding to waves from the right $\kappa=-1$.

To expand the time-domain solution in terms of the frequency-domain solutions (eigenfunctions) it is typical to introduce an orthogonality condition and to normalize the eigenfunctions based on this condition. In the case of the scattering problem Hazard \& Loret (2007) describe how the eigenfunctions satisfy the same normalization condition with and without the scatterers. It follows that the eigenfunctions satisfy the same normalization condition with and without the body and hence the contribution by the body motion can be ignored. Therefore, the normalization condition obtained in the scattering problem is also valid for the floating body problem

$$
\begin{aligned}
& \left\langle\left(\zeta_{\kappa}\left(x, k\left(\omega_{1}\right)\right) \xi_{\kappa}\left(k\left(\omega_{1}\right)\right)\right),\left(\zeta_{\kappa}^{\prime}\left(x, k\left(\omega_{2}\right)\right) \xi_{\kappa}^{\prime}\left(k\left(\omega_{2}\right)\right)\right)\right\rangle \\
= & 2 \pi \delta\left(k_{1}-k_{2}\right) \delta_{\kappa \kappa^{\prime}}=\left.2 \pi \delta_{\kappa \kappa^{\prime}} \delta\left(\omega_{1}-\omega_{2}\right) \frac{d \omega}{d k}\right|_{\omega=\omega_{1}}
\end{aligned}
$$

where it is assumed here (and hereafter) that $k$ is a function of $\omega$ and vice versa as required. Solutions of the evolution equation are expanded in terms of the frequency-domain solutions as follows

$$
\begin{aligned}
& \left(\begin{array}{c}
\zeta(x, t) \\
\xi(t)
\end{array}\right)= \\
& \int_{\mathbb{R}^{+}} \sum_{\kappa \in\{-1,1\}}\left\{f_{\kappa}(\omega) \cos (\omega t)+g_{\kappa}(\omega) \frac{\sin (\omega t)}{\omega}\right\} \times \\
& \left(\begin{array}{c}
\zeta_{\kappa}(x, k) \\
\xi_{\kappa}(k)
\end{array}\right) d \omega \quad(18)
\end{aligned}
$$

where $f_{\kappa}$ and $g_{\kappa}$ will be determined from the initial condition. The initial free-surface elevation and velocity and the body displacement and ve- locity vectors are specified as follows

$$
\left(\begin{array}{c}
\zeta(x, 0) \\
\xi(0)
\end{array}\right)=\left(\begin{array}{c}
\zeta_{0}(x) \\
\xi_{0}
\end{array}\right), \quad\left(\begin{array}{c}
\dot{\zeta}(x, 0) \\
\dot{\xi}(0)
\end{array}\right)=\left(\begin{array}{c}
\dot{\zeta}_{0}(x) \\
\mathbf{v}_{\mathbf{0}}
\end{array}\right) .
$$

Taking the inner product of the initial elevation/displacement equations with respect to a generalized eigenfunction gives

$$
\left\langle\zeta_{0}(x), \zeta_{\kappa}(x, k)\right\rangle+\xi_{0} \cdot\left(\mathbf{W} \xi_{\kappa}\right)^{*}=2 \pi f_{\kappa}(\omega) \frac{d \omega}{d k}
$$

and similarly for the velocity condition

$$
\left\langle\dot{\zeta}_{0}(x), \zeta_{\kappa}(x, k)\right\rangle+\mathbf{v}_{\mathbf{0}} \cdot\left(\mathbf{W} \xi_{\kappa}\right)^{*}=2 \pi g_{\kappa}(\omega) \frac{d \omega}{d k} .
$$

If the velocity of the free-surface and body are both initially zero then $g_{\kappa}(\omega)=0$ and the timedependent solution becomes

$$
\begin{gathered}
\left(\begin{array}{c}
\zeta(x, t) \\
\xi(t)
\end{array}\right)= \\
\frac{1}{2 \pi} \int_{\mathbb{R}^{+}} \sum_{\kappa \in\{-1,1\}}\left[\left\langle\zeta_{0}(x), \zeta_{\kappa}(x, k)\right\rangle+\xi_{0} \cdot\left(\mathbf{W} \xi_{\kappa}\right)^{*}\right] \times \\
\cos (\omega t)\left(\begin{array}{c}
\zeta_{\kappa}(x, k) \\
\xi_{\kappa}(k)
\end{array}\right) d k \quad(22)
\end{gathered}
$$

where it should be noted that that the inner product on the free-surface is explicitly defined as the integral

$$
\left\langle\zeta_{0}(x), \zeta_{\kappa}(x, k)\right\rangle=\int_{F} \zeta_{0}\left(x^{\prime}\right) \zeta_{\kappa}\left(x^{\prime}, k\right)^{*} d x^{\prime} .
$$

\section{Results}

Figure 1 shows a very simple example calculation for a body of negligible submergence which rests on the surface between $x=-1$ and $x=1$. The fluid is excited with an initial pulse and the resulting motion of the free surface and body can be seen. Further results will be presented at the workshop.

\section{Summary}

We have shown how the generalized eigenfunction method can be used to calculate the response of a floating body and have presented some example calculations. We hope that the development of this method will be useful both theoretically and practically. For example, we may be able to 

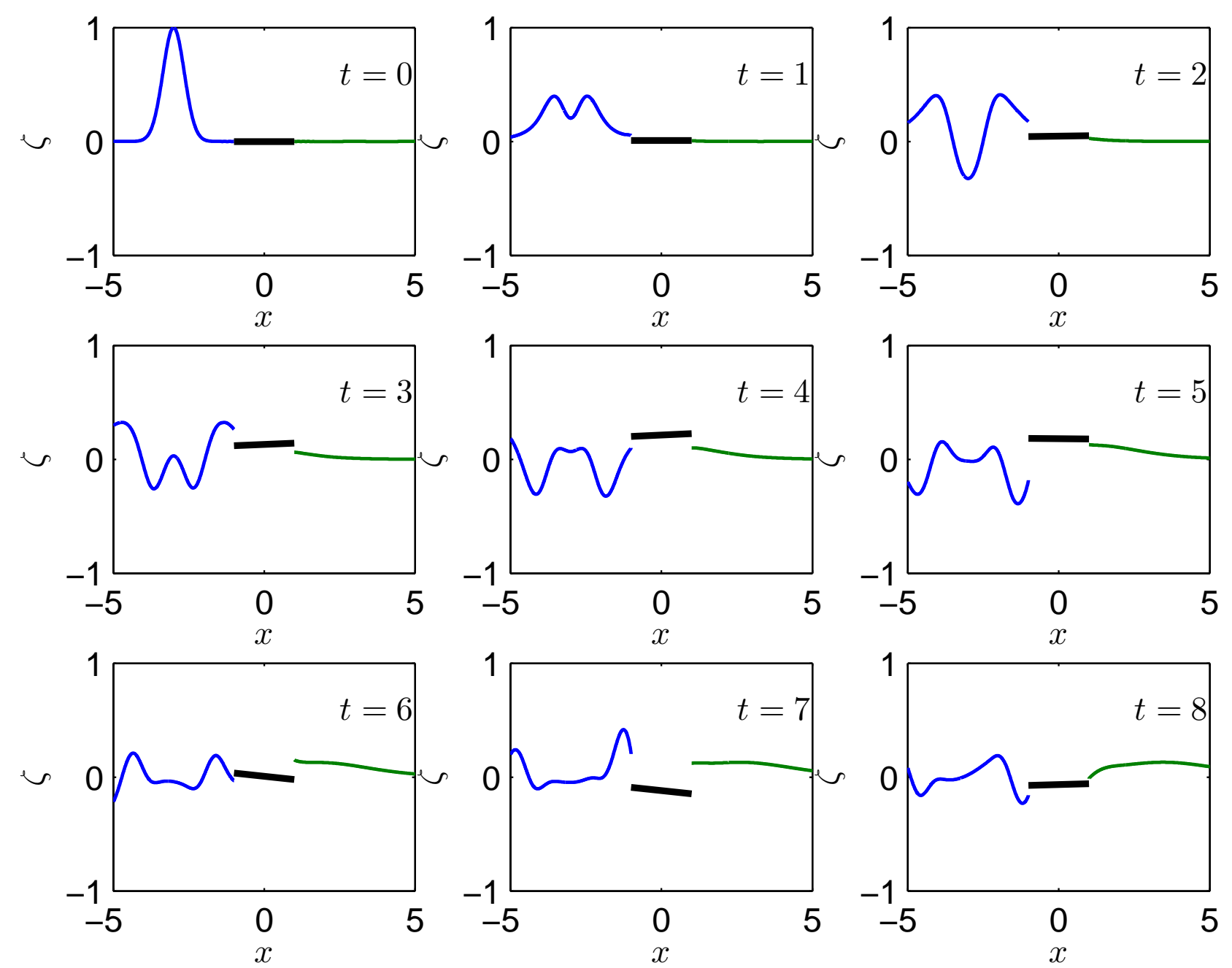

Figure 1: The response of a simple floating body by the generalized eigenfunction method.

understand the long time behaviour of a floating body as discussed in Ursell (1964); Maskell \& Ursell (1970). We also aim to make a clear connection between this method and the standard solution in the time domain by the memory effect method Mei (1989).

\section{References}

Hazard, C. \& Lenoir, M. 2002 Surface water waves. In Scattering (ed. R. P. . P. Sabatier), pp. 618-636. Academic.

Hazard, C. \& Loret, F. 2007 Generalized eigenfunction expansions for scattering problems with an application to water waves. Proc. R. Soc. Edinburgh 137A, 995-1035.

Hazard, C. \& Meylan, M. H. 2007 Spectral theory for a two-dimensional elastic thin plate floating on water of finite depth. SIAM J. Appl. Math. 68 (3), 629-647.

Maskell, S. \& URsell, F. 1970 The transient motion of a foating body. J. Fluid Mech. 44, 303-313.

Mei, C. C. 1989 The Applied Dynamics of Ocean Surface Waves. World Scientific.

Meylan, M. H. 2002 Spectral solution of time dependent shallow water hydroelasticity. $J$. Fluid Mech. 454, 387 - 402.

Meylan, M. H. 2009 Generalized eigenfunction expansion for linear water-waves. J. Fluid Mech. 632, 447-455.

Ursell, F. 1964 The decay of the free motion of a floating body. J. Fluid Mech. 19, 303-319. 